\title{
Thermodynamic, dynamic, structural, and excess entropy anomalies for core-softened potentials
}

Cite as: J. Chem. Phys. 135, 104507 (2011); https://doi.org/10.1063/1.3630941

Submitted: 21 January 2011 . Accepted: 09 August 2011 . Published Online: 13 September 2011

Ney M. Barraz, Evy Salcedo, and Marcia C. Barbosa

\section{ARTICLES YOU MAY BE INTERESTED IN}

Tetrahedral order, pair correlation entropy, and waterlike liquid state anomalies: Comparison of $\mathrm{GeO}_{2}$ with $\mathrm{BeF}_{2}, \mathrm{SiO}_{2}$, and $\mathrm{H}_{2} \mathrm{O}$

The Journal of Chemical Physics 132, 234507 (2010); https://doi.org/10.1063/1.3439593

Communication: Re-entrant limits of stability of the liquid phase and the Speedy scenario in colloidal model systems

The Journal of Chemical Physics 146, 041103 (2017); https://doi.org/10.1063/1.4974830

Thermodynamic and dynamic anomalies for a three-dimensional isotropic core-softened potential

The Journal of Chemical Physics 124, 084505 (2006); https://doi.org/10.1063/1.2168458

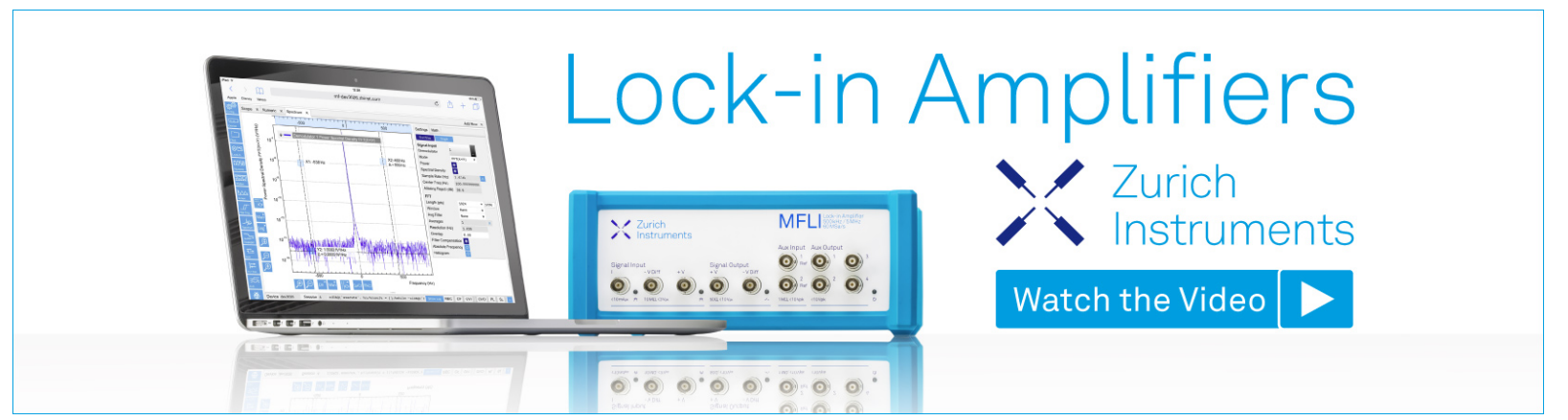

J. Chem. Phys. 135, 104507 (2011); https://doi.org/10.1063/1.3630941 


\title{
Thermodynamic, dynamic, structural, and excess entropy anomalies for core-softened potentials
}

\author{
Ney M. Barraz, Jr., ${ }^{1, a)}$ Evy Salcedo, ${ }^{2}$ and Marcia C. Barbosa ${ }^{1}$ \\ ${ }^{1}$ Instituto de Física, Universidade Federal do Rio Grande do Sul, 91501-970, Porto Alegre, RS, Brazil \\ ${ }^{2}$ Instituto de Física, Universidade Federal de Santa Catarina, 88010-970, Florianópolis, SC, Brazil
}

(Received 21 January 2011; accepted 9 August 2011; published online 13 September 2011)

\begin{abstract}
Using molecular dynamic simulations, we study three families of continuous core-softened potentials consisting of two length scales: a shoulder scale and an attractive scale. All the families have the same slope between the two length scales but exhibit different potential energy gap between them. For each family three shoulder depths are analyzed. We show that all these systems exhibit a liquid-liquid phase transition between a high density liquid phase and a low density liquid phase ending at a critical point. The critical temperature is the same for all cases suggesting that the critical temperature is only dependent on the slope between the two scales. The critical pressure decreases with the decrease of the potential energy gap between the two scales suggesting that the pressure is responsible for forming the high density liquid. We also show, using the radial distribution function and the excess entropy analysis, that the density, the diffusion, and the structural anomalies are present if particles move from the attractive scale to the shoulder scale with the increase of the temperature indicating that the anomalous behavior depends only in what happens up to the second coordination shell. (C) 2011 American Institute of Physics. [doi:10.1063/1.3630941]
\end{abstract}

\section{INTRODUCTION}

The phase behavior of single component systems as particles interacting via the so-called core-softened (CS) potentials is receiving a lot of attention recently. These potentials exhibit a repulsive core with a softening region with a shoulder or a ramp. ${ }^{1-8}$ These models originate from the desire of constructing a simple two-body isotropic potential capable of describing the complicated features of systems interacting via anisotropic potentials. This procedure generates systems that are analytically and computationally tractable and that one hopes are capable to retain the qualitative features of the real complex systems. ${ }^{9-12}$

The physical motivation behind these studies is the recently acknowledged possibility that some single component systems interacting through a core-softened potential display density and diffusion anomalies. This opened the discussion about the relation between the existence of thermodynamic anomalies in liquids and the form of the effective potential. ${ }^{13-18}$

These anomalies appear in two different ways. First, it is the density anomaly. Most liquids contract upon cooling. This is not the case of water and other fluid systems. For water, the specific volume at ambient pressure starts to increase when cooled below $T \approx 4{ }^{\circ} \mathrm{C}$. The anomalous behavior of water was first suggested 300 years ago ${ }^{19}$ and was confirmed by a number of experiments. ${ }^{9,10}$ Besides, between $0.1 \mathrm{MPa}$ and $190 \mathrm{MPa}$ water also exhibits an anomalous increase of compressibility ${ }^{20,21}$ and, at atmospheric pressure, an increase of isobaric heat capacity upon cooling..$^{22,23}$

\footnotetext{
a) Author to whom correspondence should be addressed. Electronic mail: neybarraz@gmail.com.
}

Experiments for $\mathrm{Te},{ }^{24} \mathrm{Ga}, \mathrm{Bi},{ }^{25} \mathrm{~S},{ }^{26,27}$ and $\mathrm{Ge}_{15} \mathrm{Te}_{85},{ }^{11}$ and simulations for silica, ${ }^{12,28-30}$ silicon, ${ }^{31}$ and $\mathrm{BeF}_{2},{ }^{12}$ show the same density anomaly.

But density anomaly is not the only unusual behavior that these materials have. For a normal liquid the diffusion constant, $D$, decreases under compression. This is not the case of water. $D$ increases on compression at low temperature, $T$, up to a maximum $D_{\max }(T)$ at $p=p_{D \max }(T) .{ }^{10,32}$ Numerical simulations for SPC/E (extended simple point charge) water model $^{33}$ recover the experimental results and show that the anomalous behavior of $D$ extends to the metastable liquid phase of water at negative pressure, a region that is difficult to access for experiments. ${ }^{34-37}$ In this region, the diffusivity $D$ decreases for decreasing $p$ until it reaches a minimum value $D_{\min }(T)$ at some pressure $p_{D \min }(T)$, and the normal behavior, with $D$ increasing for decreasing $p$, is re-established only for $p<p_{D \min }(T) .^{34-39}$ Besides water, silica ${ }^{28,29}$ and silicon ${ }^{40}$ also exhibit a diffusion anomalous region.

Acknowledging that CS potentials may engender density and diffusion anomalous behavior, a number of CS potentials were proposed to model the anisotropic systems described above. They possess a repulsive core that exhibits a region of softening where the slope changes dramatically. This region can be a shoulder or a ramp. ${ }^{1,6,15,16,41-59}$ These models exhibit density and diffusion anomalies, but depending on the specific shape of the potential, the anomalies might be hidden in the metastable phase. ${ }^{16}$ Also there are a number of core-softened potentials in which the anomalies are not present. ${ }^{60,61}$ The relation between the specific shape of the effective core-softened potential and the microscopic mechanism necessary for the presence of the anomalies is still under debate. ${ }^{16,57,62,63}$

Recently, it was suggested that the link between the presence of the density and diffusion anomaly and the 

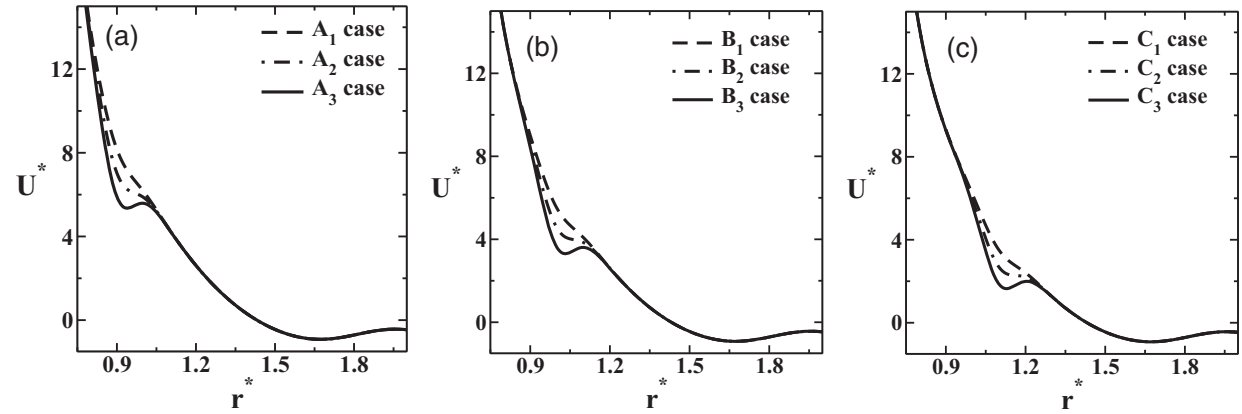

FIG. 1. Interaction potential obtained by changing parameters $h_{1}$ in the Eq. (1). The potential and the distances are in dimensionless units $U^{*}=U / \gamma$ and $r^{*}=r / r_{0}$. Here, we use $\epsilon / \gamma=0.02$ and $\sigma / r_{0}=1.47$.

microscopic details of the system can be analyzed in the framework of the excess-entropy-based formalism ${ }^{64}$ applied to similar systems by Errington et al. ${ }^{65}$ and Chakraborty and Chakravarty. ${ }^{66}$ Within this approach, the presence of the density and the diffusion anomalies is related to the density dependence of the excess entropy, $s_{\mathrm{ex}}$.

The computation of the excess entropy, however, requires integrating the radial distribution function in the whole space. The anomalous behavior, however, seems to depend only on the two length scales present in the system and, therefore, should not depend on the particle distributions far away. Here, we propose that the two length scales potentials will have density and diffusion anomalies, if the two scales would be accessible. In principle, the accessibility only depends on the distribution of particles in these two distances. Therefore, the knowledge of the complete excess entropy is not necessary for knowing if the system has or not anomalies. The behavior of the partial excess entropy computed only up to the second coordination shell should give enough information to determine if a system has anomalies or not.

In this paper, we test this assumption by computing the pressure-temperature phase diagram and the excess entropy for three families of core-softened potentials that have two length scales: a shoulder scale and an attractive scale. ${ }^{18,67}$ In all the three families, the slope between the two scales is the same. ${ }^{13}$ The shoulder scale is made more favorable by decreasing the energy gap between the two length scales (see potentials A, B, and C in Fig. 1). In addition, the shoulder scale becomes more favorable by making the depth of the shoulder scale deeper (see potentials A1, A2, and A3 in Fig. 1). The slope between the two length scales is kept fixed in order to have the liquid-liquid critical point and the density anomalous region in the same region of the pressure temperature phase diagram. ${ }^{13}$

The remaining of this paper goes as follows. In Sec. II the model is introduced. The simulations details are given in Sec. III. In Sec. IV the results are discussed. Finally, Sec. V presents the conclusion.

\section{THE MODEL}

We consider a system of $N$ particles, with diameter $\sigma$, where the pair interaction is described by a family of contin- uous potentials given by

$$
U(r)=\epsilon\left[\left(\frac{\sigma}{r}\right)^{a}-\left(\frac{\sigma}{r}\right)^{b}\right]+\sum_{j=1}^{4} h_{j} \exp \left[-\left(\frac{r-c_{j}}{w_{j}}\right)^{2}\right] .
$$

The first term is a Lennard-Jones potential and the second term is composed by four Gaussians, each Gaussian is centered in $c_{j}$. This potential can represent a whole family of intermolecular interactions, depending of the choice of the parameters $a, b, \sigma,\left\{h_{j}, c_{j}, w_{j}\right\}$, with $j=1, \ldots, 4$. The values of these parameters are in Table I. The parameters are chosen in order to obtain a two length scales potential ${ }^{67-69}$ that is related to the interaction between two tetramers. ${ }^{67,68}$

The simulations are made in dimensionless units; therefore, all the physical quantities are given in terms of the energy scale $\gamma$ and the distance scale $r_{0}$, where $\gamma$ is the energy scale and $r_{0}$ is the length scale chosen so the minimum of the potential in the $B_{3}$ case is about $r^{*}=1$. Here, we use $\epsilon / \gamma=0.02$ and $\sigma / r_{0}=1.47$.

Modifying the parameters $c_{1}$ and $h_{1}$ in Eq. (1), according to Table II, allow us to change the depth of the shoulder well as illustrated in Fig. 1. Here, we use nine different values for $h_{1}$ and they are expressed as a multiple of a reference value $h_{1}^{\text {ref }}$. We also use three different values of $c_{1}$ and they are expressed as a multiple of a reference value $c_{1}^{\text {ref }}$. For all the nine cases, the values of $a, b,\left\{w_{j}\right\}$ with $j=1, \ldots, 4$, $c_{i}$ with $i=2, \ldots, 3, h^{r e f}$, and $c^{\text {href }}$ are fixed and given by Table I. Table III gives the parameter values in $\AA$ and $\mathrm{kcal} / \mathrm{mol}$ consistent with modeling ST4 water. ${ }^{67}$

Modifying the distance between the two minima of the two scales, shoulder scale and attractive scale, leads to the three families $A, B$ and $C$ as shown in Fig. 1. The changes in the distance between the two length scales were done in such way to preserve the slope between the two scales and,

TABLE I. Parameters for potentials $A, B$, and $C$ in reduced unit.

\begin{tabular}{lcccccccc}
\hline Parameter & Value & Parameter & \multicolumn{2}{c}{ Value } & Parameter & Value & Parameter & Value \\
\hline$a$ & 9.056 & $w_{1}$ & 0.085 & $c_{1}^{\text {ref }}$ & 0.996 & $h_{1}^{\text {ref }}$ & -3.79 \\
$b$ & 4.044 & $w_{2}$ & 0.618 & $c_{2}$ & 0.529 & $h_{2}$ & 1.209 \\
$\epsilon$ & 0.020 & $w_{3}$ & 0.826 & $c_{3}$ & 1.598 & $h_{3}$ & -1.503 \\
$\sigma$ & 1.475 & $w_{4}$ & 0.214 & $c_{4}$ & 1.929 & $h_{4}$ & 0.767 \\
\hline
\end{tabular}


TABLE II. Parameters of $c_{1}$ and $h_{1}$ for potentials $A, B$, and $C$.

\begin{tabular}{lccccccc}
\hline \hline Potentials & Values & Potentials & Values & Potentials & Values & Potentials & Values \\
\hline$A$ & $0.90 c_{1}^{\text {ref }}$ & $A_{1}$ & $0.30 h_{1}^{\text {ref }}$ & $B_{1}$ & $0.25 h_{1}^{\text {ref }}$ & $C_{1}$ & $0.22 h_{1}^{\text {ref }}$ \\
$B$ & $1.00 c_{1}^{\text {ref }}$ & $A_{2}$ & $0.60 h_{1}^{\text {ref }}$ & $B_{2}$ & $0.50 h_{1}^{\text {ref }}$ & $C_{2}$ & $0.44 h_{1}^{\text {ref }}$ \\
$C$ & $1.10 c_{1}^{\text {ref }}$ & $A_{3}$ & $0.90 h_{1}^{\text {ref }}$ & $B_{3}$ & $0.75 h_{1}^{\text {ref }}$ & $C_{3}$ & $0.66 h_{1}^{\text {ref }}$ \\
\hline \hline
\end{tabular}

therefore, to have in all the cases the region of density anomaly in the same region of the pressure-temperature phase diagram as proposed by Yan et al. ${ }^{70}$

The family $A$ has the largest distance and the largest potential energy gap between the two length scales $\left(a_{A}=0.72\right.$ in Fig. 2), the family $B$ has the intermediate distance and intermediate potential energy gap between the two length scales $\left(a_{B}=0.62\right.$ in Fig. 2), and the family $C$ has the shortest distance and the smallest potential energy gap between the two length scales, $\left(a_{C}=0.52\right.$ in Fig. 2).

For each family, we analyze three different depths of the shoulder scale represented by 1, 2, and 3 (see Fig. 2). The potentials 1 have the most shallow shoulder scale, the potentials 2 have intermediate shoulder depth, and the potentials 3 have the deepest shoulder scale. Table III gives the values for the depths for each one of the families.

In summary, we analyze nine different potentials: $A_{1}, A_{2}, A_{3}, B_{1}, B_{2}, B_{3}, C_{1}, C_{2}$, and $C_{3}$ as illustrated in Fig. 1. The values of the different $h_{1}$ for each case are listed in Table II.

Barraz et al. ${ }^{18}$ investigated the family $B$. It was shown that this potential exhibits thermodynamic, dynamic, and structural anomalies if the shoulder scale is not too deep. Their result suggests that in order to have anomalies it is necessary but not sufficient to have two length scales competing. Both length scales must be accessible. When the shoulder scale becomes too deep, the particles are trapped in this length scale and no anomaly is present. By making the shoulder deeper, we are decreasing the difference in energy between the scales and, therefore, destroying the competition.

Here, we explore the accessibility or the absence of accessibility by changing both the energy gap between the two length scales and the distance between them.

\section{THE SIMULATION DETAILS}

The properties of the system were obtained by $N V T$ molecular dynamics using Nose-Hoover heat-bath with coupling parameter $Q=2$. The system is characterized by 500 particles in a cubic box with periodic boundary conditions, interacting with the intermolecular potential described above. All physical quantities are expressed in reduced units ${ }^{71}$ (the

TABLE III. Values for the depths for each one of the families.

\begin{tabular}{lccccc}
\hline \hline Potential & Value & Potential & Value & Potential & Value \\
\hline$b_{A_{1}}$ & 7.10 & $b_{B_{1}}$ & 4.94 & $b_{C_{1}}$ & 2.95 \\
$b_{A_{2}}$ & 6.20 & $b_{B_{2}}$ & 4.07 & $b_{C_{2}}$ & 2.33 \\
$b_{A_{3}}$ & 5.28 & $b_{B_{3}}$ & 3.32 & $b_{C_{3}}$ & 1.60 \\
\hline \hline
\end{tabular}

units of length and energy are, respectively, $r_{0}$ and $\gamma$ ) and are given by

$$
\begin{aligned}
t^{*} & =t r_{0}(m / \gamma)^{1 / 2}, \\
T^{*} & =\frac{k_{B} T}{\gamma}, \\
p^{*} & =\frac{p r_{0}^{3}}{\gamma}, \\
\rho^{*} & =\rho r_{0}^{3}, \\
D^{*} & =D \sqrt{\frac{m}{\gamma r_{0}^{2}}} .
\end{aligned}
$$

Standard periodic boundary conditions together with predictor-corrector algorithm were used to integrate the equations of motion with a time step $\Delta t^{*}=0.002$ and potential cut off radius $r_{c}^{*}=3.5$. The initial configuration were set on solid and on liquid states and, in both cases, the equilibrium state was reached after $t_{e q}^{*}=1000$ (what is in fact 500000 steps since $\Delta t^{*}=0.002$ ). From this time on, the physical quantities were stored in intervals of $\Delta t_{R}^{*}=1$ during $t_{R}^{*}=1000$. The system is uncorrelated after $t_{d}^{*}=10$, from the velocity auto-correlation function. With 50 descorrelated samples were used to get the average of the physical quantities.

The thermodynamic stability of the system was checked by analyzing the dependence of pressure on density, namely,

$$
\frac{\partial p}{\partial \rho}<0,
$$

by the behavior of the energy and also by visual analysis of the final structure, searching for cavitation.

At the phase boundary between the liquid and the amorphous phases we found stable states points at both phases. The state with the lower energy was considered. In this particular
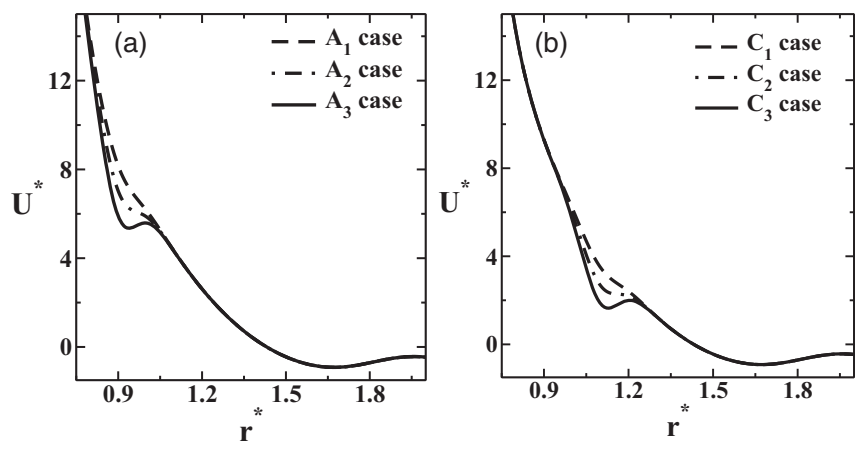

FIG. 2. Scheme to distinguish the distance between the scales and depths of the first scale. 
TABLE IV. First critical point location for potentials $A, B$, and $C$.

\begin{tabular}{lcccccccc}
\hline \hline Potential & $T_{c 1}^{*}$ & $p_{c 1}^{*}$ & Potential & $T_{c 1}^{*}$ & $p_{c 1}^{*}$ & Potential & $T_{c 1}^{*}$ & $p_{c 1}^{*}$ \\
\hline$A_{1}$ & 1.94 & 0.074 & $B_{1}$ & 1.93 & 0.072 & $C_{1}$ & 1.98 & 0.076 \\
$A_{2}$ & 1.95 & 0.074 & $B_{2}$ & 1.98 & 0.078 & $C_{2}$ & 2.08 & 0.088 \\
$A_{3}$ & 1.97 & 0.076 & $B_{3}$ & 2.02 & 0.080 & $C_{3}$ & 2.20 & 0.099 \\
\hline \hline
\end{tabular}

region of the pressure-temperature phase diagram, the energy was a good approximation for the Helmoltz free energy.

The error bars for temperatures and pressures away from the critical region are smaller than the size of the gray lines. The error bar near the critical point are $\Delta T=0.0025$ and $\Delta p=0.05$. Our error is controlled by making averages of uncorrelated measures.

\section{RESULTS}

\section{A. Pressure-temperature phase diagram}

First, we explore the effects that the increase of the shoulder depth and the decrease of the distance between the two scales have in the location in the pressure-temperature phase diagram of the different phases. Figure 3 illustrates the pressure versus temperature phase diagram of the three families $A, B$, and $C$ of potentials. At high temperatures there are a fluid phase and a gas phase (not shown). These two phases coexist at a first order line that ends at a critical point (see Table IV for the pressure and the temperature values).

At low temperatures and high pressures, there are two liquid phases coexisting at a first order line ending at a second critical point (see Table $\mathrm{V}$ for the pressure and the temperature values). The thermodynamic stability of the state points was checked by analyzing the dependence of pressure on density using Eq. (3). The critical point was identified in the graph by the region where isochores cross. The coexistence line was obtained as the medium line between the stability limit of each phase.

Comparison between the cases $A_{1}, B_{1}$, and $C_{1}$ indicates that as the distance between the two scales decreases, the critical pressure decreases but the critical temperature remains the same as illustrated in Fig. 4. This observation is confirmed in the cases $A_{2}, B_{2}$, and $C_{2}$ and in the cases $A_{3}, B_{3}$, and $C_{3}$ (see Table $\mathrm{V}$ for the critical pressures and the temperatures).

How can this result be understood? The two liquid phases are formed due to the presence of the two competing scales. The low density liquid is related to the attractive scale while the high density liquid is related to the shoulder scale. In order to reach the high density liquid phase, the system has to overcome a large potential energy but also have to become very compact in the case $A_{1}$. The potential energy gap and the dis-

TABLE V. Second critical point location for potentials $A, B$, and $C$.

\begin{tabular}{lcccccccr}
\hline \hline Potential & $T_{c 2}^{*}$ & $p_{c 2}^{*}$ & Potential & $T_{c 2}^{*}$ & $p_{c 2}^{*}$ & Potential & $T_{c 2}^{*}$ & $p_{c 2}^{*}$ \\
\hline$A_{1}$ & 0.34 & 4.21 & $B_{1}$ & 0.35 & 3.44 & $C_{1}$ & 0.34 & 2.59 \\
$A_{2}$ & 0.48 & 3.16 & $B_{2}$ & 0.48 & 1.86 & $C_{2}$ & 0.41 & 0.78 \\
$A_{3}$ & 0.59 & 1.70 & $B_{3}$ & 0.57 & 0.49 & $C_{3}$ & 0.53 & -0.63 \\
\hline \hline
\end{tabular}

tance between the two length scales are higher in the case $A$ than in the case $C$; therefore, the pressure needed for forming a high density liquid phase is higher in the case $A$ than it is in the case $C$.

At very low temperatures, the system becomes less diffusive and crystallization might be expected. Here, we do not explore all the possible crystal phases, but instead as the temperature is decreased from the liquid phase an amorphous phase is formed. The dotted line in Fig. 3 shows the separation between the fluid phase and the amorphous region. The amorphous phase is identified by the region where the diffusion coefficient becomes zero and the radial distribution functions do not exhibit the periodicity of a solid. Table VI have the characteristic pressure and temperature values of the amorphous phase boundary for the different models. It shows that the region in the pressure-temperature phase diagram, where the amorphous phase is present shrinks as the shoulder part of the potential becomes deeper.

\section{B. Density anomaly}

Next, we test the effects that the decrease of the distance between the two length scales and the increase of the depth of the first scale have in the presence of the density anomaly. Figure 3 shows the isochores $0.30 \leq \rho^{*} \leq 0.65$ represented by thin solid lines for the nine models. Equation

$$
\left(\frac{\partial V}{\partial T}\right)_{p}=-\left(\frac{\partial p}{\partial T}\right)_{V}\left(\frac{\partial V}{\partial p}\right)_{T}
$$

indicates that the temperature of minimum pressure at constant density is the temperature of maximum density at constant pressure, the TMD. The TMD is the boundary of the region of thermodynamic anomaly, where a decrease in the temperature at constant pressure implies an anomalous increase in the density and, therefore, an anomalous behavior of density (similar to what happens in water). Figure 3 show the TMD as solid thick lines. For all potentials $A, B$, and the potentials $C_{1}$ and $C_{2}$, the TMD is present but for potential $C_{3}$ the TMD is not observed.

Similar to what happens with the location of amorphous region, as the distance between the shoulder and the attractive scales decreases, the region in the pressure-temperature phase diagram delimited by the TMD line shrinks and disappears for the case $C_{3}$. For the potential $B$, the TMD line is located at temperatures below the temperature of the liquid-liquid critical point. The thermodynamic parameters that limit the TMD in phase diagram are shown in Table VII, where $p_{l}$ represents the values of $\left(\rho^{*}, T^{*}, p^{*}\right)$ for the point of the lowest pressure in the TMD line, $p_{m}$ is the point with the highest temperature and $p_{h}$ is the point with the highest pressure.

How can this result be understood? The density anomalous behavior arises from the competition between the two length scales: the shoulder and attractive scales. At high pressures, the shoulder scale wins and at low pressures the attractive scale wins. The density anomalous region exists only in the intermediate pressure range where clusters of both scales are present. The value of the "high" pressure and of the "low" pressure is determined by the difference in energy between 



FIG. 3. Pressure-temperature phase diagram for the family $A$ in the left-hand side, for the family $B$ in the middle, and for $C$ in the right-hand side. The thin solid lines are the isochores $0.30<\rho^{*}<0.65$. The liquid-liquid critical point is the dot, the temperature of maximum density is the solid thick line, the diffusion extrema is the dashed line, and the structural extrema is the dashed-dotted line. The dotted line indicates the limit between the fluid and the amorphous regions. For the diffusion coefficient and radial distribution functions, it is possible to determine the amorphous region. The full line on the left side of the second critical point is an approximate line of the coexistence of fluids of high and low density.
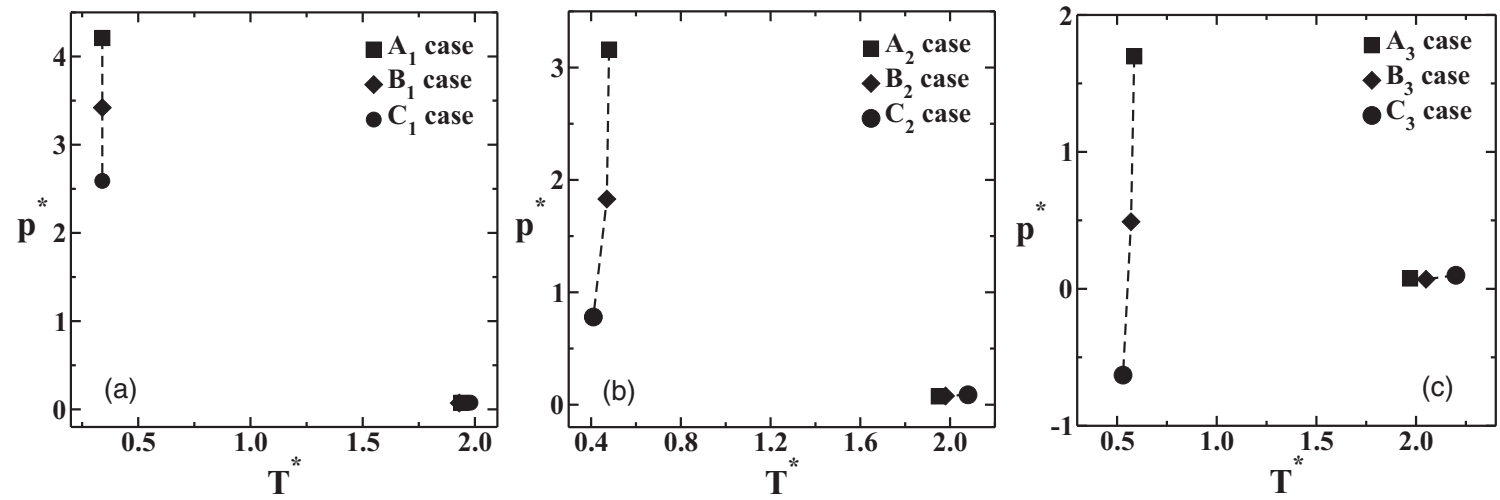

FIG. 4. Location of the critical points on pressure-temperature phase diagram for cases $A, B$, and $C$. 
TABLE VI. Values of pressure location in the amorphous region for cases $A, B$, and $C$.

\begin{tabular}{lccccc}
\hline \hline Potentials & Values & Potentials & Values & Potentials & Values \\
\hline$A_{1}$ & $-0.90 \lesssim p^{*} \lesssim 3.70$ & $B_{1}$ & $-0.91 \lesssim p^{*} \lesssim 3.40$ & $C_{1}$ & $-0.85 \lesssim p^{*} \lesssim 2.35$ \\
$A_{2}$ & $-0.30 \lesssim p^{*} \lesssim 2.80$ & $B_{2}$ & $-0.89 \lesssim p^{*} \lesssim 1.80$ & $C_{2}$ & $-0.76 \lesssim p^{*} \lesssim 0.55$ \\
$A_{3}$ & $-1.05 \lesssim p^{*} \lesssim 1.53$ & $B_{3}$ & $-1.00 \lesssim p^{*} \lesssim 0.48$ & $C_{3}$ & $\ldots$ \\
\hline \hline
\end{tabular}

the two scales. If the difference is too small the low and high pressures are too close and no anomaly appears.

\section{Diffusion anomaly}

Then, we check the effects that the decrease of the distance between the two scales have in the location in the pressure-temperature phase diagram of the diffusion anomaly. The diffusion coefficient is obtained from the expression

$$
D=\lim _{t \rightarrow \infty} \frac{\left\langle\left[\vec{r}_{j}\left(t_{0}+t\right)-\vec{r}_{j}\left(t_{0}\right)\right]^{2}\right\rangle_{t_{0}}}{6 t}
$$

where $\vec{r}_{j}(t)$ are the coordinates of particle $j$ at time $t$, and $\langle\cdots\rangle_{t_{0}}$ denotes an average over all particles and over all $t_{0}$.

Figure 5 shows the behavior of the dimensionless translational diffusion coefficient, $D^{*}$, as function of the dimensionless density, $\rho^{*}$, at constant temperature for the cases: $A_{3}, B_{3}$, and $C_{3}$. The solid lines are a polynomial fits to the data obtained by simulation (the dots in Fig. 5). For normal liquids, the diffusion at constant temperature increases with the decrease of the density. For the cases $A_{1}, A_{2}, B_{1}, B_{2}, B_{3}, C_{1}, C_{2}$ (not shown) and for the cases $A_{3}$ and $B_{3}$ the diffusion has a region in the pressure-temperature phase diagram in which the diffusion increases with density. This is the diffusion anomalous region illustrated in Fig. 3 as a dashed line.

Similar to what happens with the location of the TMD, as the two length scales become closer, the region in the pressure-temperature phase diagram delimited by the extrema of the diffusion goes to lower pressures, shrinks, and disappears for the case $C_{3}$.

Figure 3 illustrates that the region in the pressuretemperature phase diagram where the dynamic anomaly occurs englobes the region where the thermodynamic anomaly is present. This hierarchy between the anomalies is observed in simulations ${ }^{34,35,57}$ and in experiments ${ }^{10}$ for bulk water.

\section{Structural anomaly}

Now, we test the effects that the decrease of the distance between the two length scales have in the location in the pressure-temperature phase diagram of the structural anomalous region.

The translational order parameter is defined as ${ }^{29,35,72}$

$$
t=\int_{0}^{\xi_{c}}|g(\xi)-1| d \xi
$$

where $\xi=r \rho^{1 / 3}$ is the distance $r$ in units of the mean interparticle separation $\rho^{-1 / 3}, \xi_{c}$ is the cutoff distance set to half of the simulation box times ${ }^{55} \rho^{-1 / 3}, g(\xi)$ is the radial distribution function which is proportional to the probability of finding a particle at a distance $\xi$ from a referent particle. The translational order parameter measures how structured is the system. For an ideal gas it is $g=1$ and $t=0$, while for the crystal phase it is $g \neq 1$ over long distances resulting in a large $t$. Therefore for normal fluids $t$ increases with the increase of the density.

Figure 6 shows the translational order parameter as a function of the density for fixed temperatures for potentials $A_{3}, B_{3}$, and $C_{3}$. The dots represent the simulation data and the solid line, the polynomial fit to the data. For the potentials $A_{3}$ and $B_{3}$, there are a region of densities in which the translational parameter decreases as the density increases. A dotteddashed line illustrates the region of local maximum and minimum of $t^{*}$ limiting the anomalous region. For the potential $C_{3}, t^{*}$ increases with the density. No anomalous behavior is observed. The potentials $A_{1}, A_{2}, B_{1}, B_{2}, C_{1}$, and $C_{2}$ that do show anomalous behavior are not shown here for simplicity.

Figure 3 shows the structural anomaly for the families $A$, $B$, and $C$, as dotted-dashed lines. It is observed that the region of structural anomaly embraces both dynamic and thermodynamic anomalies. As the distance between the shoulder and

TABLE VII. Limiting values for density $\left(\rho^{*}\right)$, temperature $\left(T^{*}\right)$, and pressure $\left(p^{*}\right)$ of the thermodynamics anomalies on pressure-temperature diagram. Here, the point $p_{l}$ represents the density, temperature, and pressure of the point of the lowest pressure in the TMD line, $p_{m}$ represents the point of the highest

\begin{tabular}{|c|c|c|c|c|c|c|c|c|c|c|c|c|c|c|}
\hline Cases & & $p_{l}$ & $p_{m}$ & $p_{h}$ & Cases & & $p_{l}$ & $p_{m}$ & $p_{h}$ & Cases & & $p_{l}$ & $p_{m}$ & $p_{h}$ \\
\hline & $\rho^{*}$ & 0.47 & 0.54 & 0.61 & & $\rho^{*}$ & 0.47 & 0.52 & 0.57 & & $\rho^{*}$ & 0.45 & 0.51 & 0.55 \\
\hline \multirow[t]{3}{*}{$A_{1}$} & $T^{*}$ & 0.71 & 1.00 & 0.72 & $B_{1}$ & $T^{*}$ & 0.71 & 0.85 & 0.69 & $C_{1}$ & $T^{*}$ & 0.56 & 0.69 & 0.40 \\
\hline & $p^{*}$ & 1.74 & 3.22 & 4.50 & & $p^{*}$ & 1.50 & 2.50 & 3.30 & & $p^{*}$ & 0.79 & 1.96 & 2.60 \\
\hline & $\rho^{*}$ & 0.46 & 0.51 & 0.59 & & $\rho^{*}$ & 0.46 & 0.50 & 0.54 & & $\rho^{*}$ & 0.46 & 0.48 & 0.50 \\
\hline \multirow[t]{3}{*}{$A_{2}$} & $T^{*}$ & 0.70 & 1.00 & 0.54 & $B_{2}$ & $T^{*}$ & 0.67 & 0.76 & 0.63 & $C_{2}$ & $T^{*}$ & 0.49 & 0.53 & 0.48 \\
\hline & $p^{*}$ & 1.36 & 2.20 & 3.17 & & $p^{*}$ & 0.90 & 1.40 & 1.80 & & $p^{*}$ & 0.23 & 0.45 & 0.64 \\
\hline & $\rho^{*}$ & 0.45 & 0.48 & 0.54 & & $\rho^{*}$ & 0.40 & 0.42 & 0.43 & & $\rho^{*}$ & $\ldots$ & $\ldots$ & $\ldots$ \\
\hline \multirow[t]{2}{*}{$A_{3}$} & $T^{*}$ & 0.66 & 0.89 & 0.69 & $B_{3}$ & $T^{*}$ & 0.44 & 0.54 & 0.52 & $C_{3}$ & $T^{*}$ & $\ldots$ & $\ldots$ & $\ldots$ \\
\hline & $p^{*}$ & 0.91 & 1.27 & 1.72 & & $p^{*}$ & 0.15 & 0.29 & 0.36 & & $p^{*}$ & $\ldots$ & $\ldots$ & $\ldots$ \\
\hline
\end{tabular}
temperature and $p_{h}$ represents the point of the highest pressure of the TMD line. 

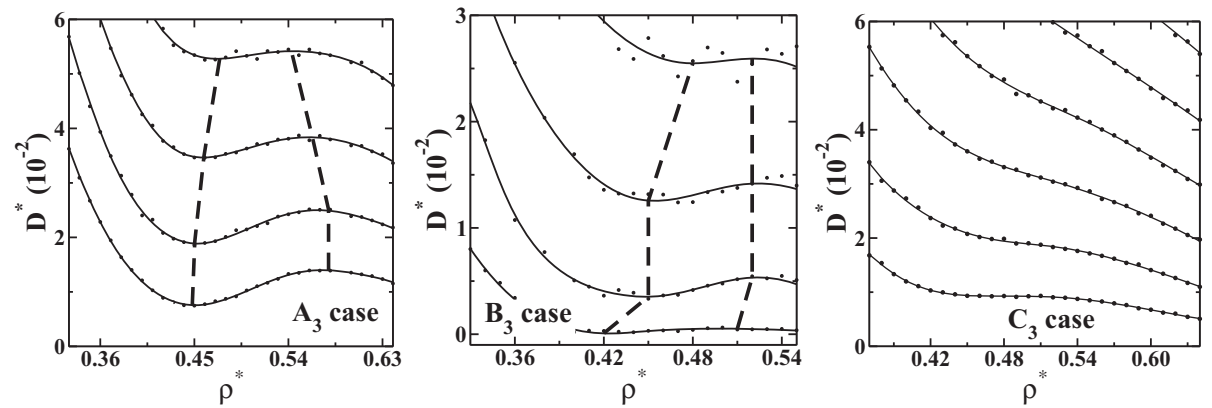

FIG. 5. Diffusion coefficient versus reduced density for $T^{*}=0.6,0.7,0.8,0.9$ from the bottom to the top for the case $A_{3}, T^{*}=0.4,0.5,0.6,0.7$ from the bottom to the top for the case $B_{3}$, and $T^{*}=0.5,0.6,0.7,0.8,0.9$ from the bottom to the top for the case $C_{3}$. The dots are the simulational data and the solid lines are polynomial fits. The dashed lines connect the densities of minima and maxima diffusivity that limit the diffusion anomalous region.

attractive scales is decreased, the structural anomalous region in the pressure-temperature phase diagram shrinks.

Another measure of the anomalous behavior is the orientational order parameter ${ }^{73} Q_{6}$. This parameter is used to get information about tetrahedral order of the molecules. For two length scales, ${ }^{76}$ spherical symmetric continuous (continuous force) potentials, $Q_{6}{ }^{55,74}$ exhibit a region of temperatures in which it decreases with increasing density. The maximum of $Q_{6}$ is located in the same region in the pressure temperature phase diagram of the maximum of the translational order parameter. ${ }^{55}$ A similar behavior is expected for our potential.

In resume, for all the density, diffusion and structural anomalous regions in the pressure-temperature phase diagram, as the two length scales become closer the region of the pressure-temperature phase diagram occupied by the anomalous region shrinks. The same effect is observed when the shoulder scale becomes deeper. ${ }^{18}$

\section{E. Radial distribution function}

What is the origin of the disappearance of the thermodynamic, dynamic, and structural anomalous behavior with the decrease of the distance between the scales? In order to answer this question the behavior of the radial distribution function for the nine different potentials is studied. The radial distribution function is a measure of the probability of finding a pair of atoms separated by $r$. This function is defined as

$$
g(r)=\frac{V}{N^{2}}\left\langle\sum_{i, j=1}^{N} \delta\left[\vec{r}-\left(\vec{r}_{i}\left(t_{0}+t\right)-\vec{r}_{j}\left(t_{0}\right)\right)\right]\right\rangle_{t_{0}},
$$

where $\vec{r}_{j}(t)$ are the coordinates of particle $i$ and $j$ at time $t$, $V$ is volume of system, $N$ is number of particles and $\langle\cdots\rangle_{t}$ denotes an average over all particles.

Recently, it was shown that a necessary condition for the presence of density anomaly is to have particles moving from one scale to the other as the temperature is increased, ${ }^{55,57,63}$ for a fixed density. Here, we test if this assumption is confirmed in the potentials we are analyzing. Figure 7 illustrates the radial distribution function versus distance for a fixed density and various temperatures for the potentials $A_{3}, B_{3}$, and $C_{3}$. For the potentials $A_{3}$ and $B_{3}$, the percentage of particles in the first length scale increases while the percentage of particles in the second length scale decreases as the temperature is increased. This means that as the system is heated at constant density particles move from one scale to the other. This behavior is also observed for the potentials $A_{1}, A_{2}, B_{1}, B_{2}, C_{1}$, and $C_{2}$ (not shown here for simplicity) and confirms our assumption that the presence of anomalies is related with particles moving from one length scale to the other length scale. ${ }^{55,57}$ This is not the case for the potential $C_{3}$. For the case $C_{3}$, as the temperature is increased the particles move from the second to the other further away coordination shells and the percentage of particles at the first scale is not affected by
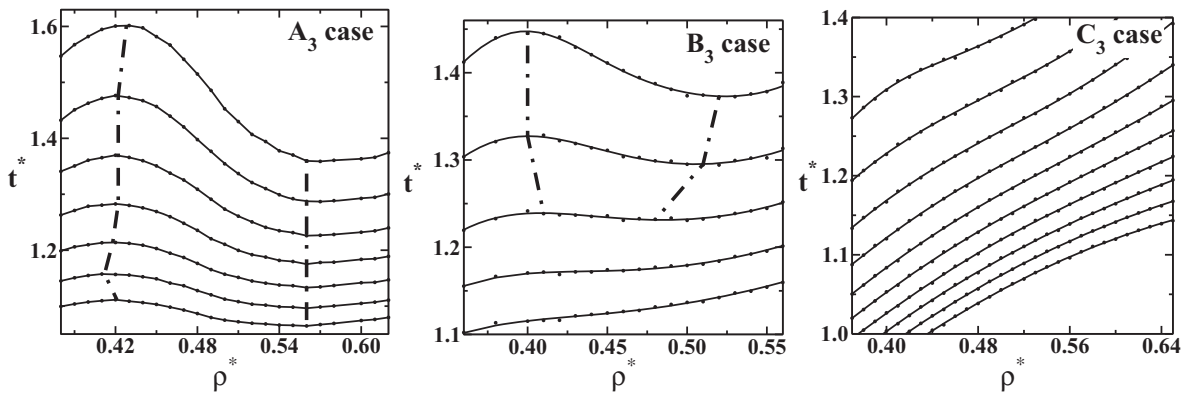

FIG. 6. The translational order parameter as a function of density for fixed temperatures: $T^{*}=1.50,1.40,1.30,1.20,1.10,1.00,0.90,0.80,0.70$, and 0.60 (from top to bottom) for the setting potentials $A$ and $C$; and $T^{*}=1.00,0.90,0.80,0.70$, and 0.60 for the setting potential $B$. The dotted-dashed lines locate the density of maxima and minima $t^{*}$. 



FIG. 7. Radial distribution function versus reduced distance for three cases, $A_{3}, B_{3}, C_{3}$ for reduced density $\rho^{*}=0.480,0.420$, and 0.390 , respectively. In cases $A_{3}$ and $B_{3}$ the first peak of $g\left(r^{*}\right)$ increases with increasing temperature, while the second peak decreases. In $C_{3}$ case for the first peak potential keeps constant while the second peak decreases.

the increase in temperature and, therefore, no anomaly is observed. ${ }^{55,57}$

How can we understand this result? The density anomaly appears if particles move from the second length scale to the first length scale. In the case of the potentials $A_{3}$ and $B_{3}$, the difference in energy between the two scales is high and heat is required for having particles reaching the shoulder length scale. Consequently, as the temperature is increased at constant density, more particles will be at the first scale and pressure decreases (see Fig. 7). In the potential $C_{3}$, the difference in energy between the two length scales is small. Almost no heat is required to have particles in the first length scales that saturate. So particles actually do not move from one scale to the other.

This picture in terms of the presence of particles in the different shells can also be checked in the framework of the excess entropy. ${ }^{55,57}$

\section{F. Excess entropy and anomalies}

The excess entropy is defined as the difference between the entropy of the real fluid and that of an ideal gas at the same temperature and density. It also can be given by its two-body contribution of $s_{\mathrm{ex}}$,

$$
s_{\mathrm{sex}} \approx s_{2}=-2 \pi \rho \int_{0}^{\infty}[g(r) \ln g(r)-g(r)+1] r^{2} d r,
$$

gives a good approximation of $s_{\mathrm{sex}}$.
What can we learn from the excess entropy about the mechanism responsible for the density, the diffusion, and the structural anomalies? In Sec. IV E we have shown, using the radial distribution function, that for potentials that exhibit density, diffusion, and structural anomalous behavior as the temperature is increased particles move from the second coordination shell to the first coordination shell. In the case of systems in which no anomalies are present, as the temperature is increased particles will move from the first and second shells to further shells. Therefore, in principle the information about the behavior of particles up to the second coordination shell would be enough to predict if a system would have anomalous behavior.

In order to test this assumption, we compute the integrals in the expression for $s_{2}^{(2)}$ (see Eq. (8)) up to the second coordination shell, namely,

$$
s_{2}^{(2)}=-2 \pi \rho \int_{0}^{r_{2}}[g(r) \ln g(r)-g(r)+1] r^{2} d r,
$$

where $r_{2}$ is the distance of the second shell.

Figure 8 shows the density dependence of $s_{2}$ along a series of isotherms spanning from $T^{*}=0.60$ to $T^{*}=1.50$ for the cases $A_{3}, B_{3}$, and $C_{3}$.

Figure 9 shows the density dependence of $s_{2}^{(2)}$ along a series of isotherms spanning from $T^{*}=0.60$ to $T^{*}=1.50$ for the cases $A_{3}, B_{3}$, and $C_{3}$. The dots are the simulational data and the solid lines are polynomial fits.
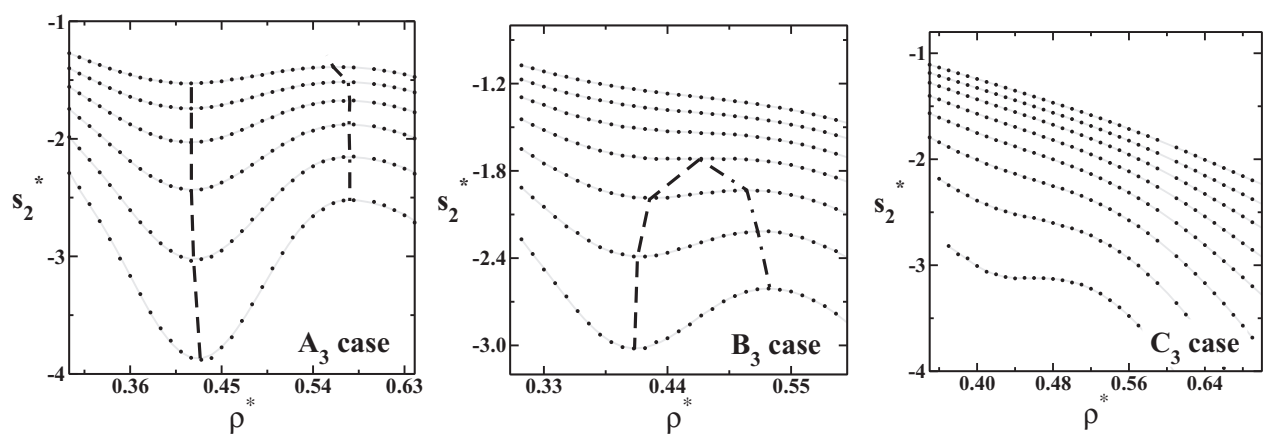

FIG. 8. Excess entropy, $s_{2}$, versus reduced density for fixed temperatures $T^{*}=0.6,0.7,0.8,0.9,1.0,1.1$ from the bottom to the top for the case $A_{3}, T^{*}$ $=0.6,0.7,0.8,0.9,1.0,1.1,1.2$ from the bottom to the top for the case $B_{3}$, and temperatures $T^{*}=0.5,0.6,0.7,0.8,0.9,1.0,1.1,1.2$ from the bottom to the top for the case $C_{3}$. The temperature $T^{*}=0.5$ contains densities that are metastable points regarding the high density liquid phase. The dotted-dashed lines locate the density of maxima $s_{2}$ and the dashed, the minima. 

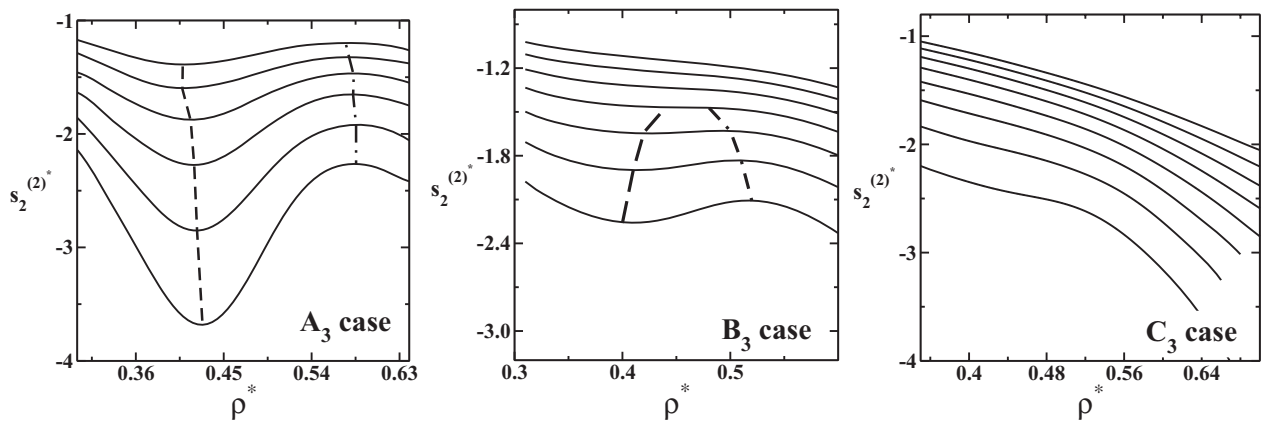

FIG. 9. Excess entropy computed up to the second coordination shell, $s_{2}^{2}$, versus reduced density for fixed temperatures $T=0.6,0.7,0.8,0.9,1.0,1.1$ from the bottom to the top for the case $A_{3}, T=0.6,0.7,0.8,0.9,1.0,1.1,1,2$ from the bottom to the top for the $B_{3}$ and temperatures, $T=$ $0.5,0.6,0.7,0.8,0.9,1.0,1.1,1.2$ from the bottom to the top for the case $C_{3}$. The temperature $T^{*}=0.5$ contains densities that are metastable points regarding the high density liquid phase. The dotted-dashed lines locate the density of maxima $s_{2}$ and the dashed, the minima.

Notice that both $s_{2}^{(2)}$ and $s_{2}$ has a maximum and a minimum for the cases $A_{3}$ and $B_{3}$ but not for the case $C_{3}$ what indicates an anomalous behavior in the excess entropy. Comparison between Figs. 8 and 9 shows that the excess entropy computed up to the second shell not only gives the same trend but also the same density for the maximum and minimum of the excess entropy.

Errington et al. have shown that the density anomaly is given by the condition $\Sigma_{\mathrm{ex}}=\left(\partial s_{\mathrm{ex}} / \partial \ln \rho\right)_{T}>1 .{ }^{65}$ They have also suggested that the diffusion anomaly can be predicted by using the empirical Rosenfeld's parametrization. ${ }^{75}$ Based on Rosenfeld's scaling parameters and approximating the excess entropy and its derivative by the two-body contribution, namely, $s_{\mathrm{ex}} \approx s_{2}$ and $\Sigma_{\mathrm{ex}}=\Sigma_{2}$ the anomalous behavior of the thermodynamic and dynamic quantities are observed if

$$
\begin{aligned}
\text { excess entropy } & \rightarrow \sum_{2} \geq 0, \\
\text { diffusivity } & \rightarrow \sum_{2} \geq 0.42, \\
\text { viscosity } & \rightarrow \sum_{2} \geq 0.83, \\
\text { density } & \rightarrow \sum_{2} \geq 1.00 .
\end{aligned}
$$

This sequence of anomalies is consistent with the studies of Yan et al., ${ }^{15,57,61-63,74}$ where structural anomalies are found to precede diffusivity anomalies, which in turn precede density anomalies.
In order to check these criteria in our family of potentials, we compute $\sum_{2}$ given by

$$
\sum_{2}=\left(\frac{\partial s_{2}}{\partial \ln \rho}\right)_{T}=s_{2}-2 \pi \rho^{2} \int \ln g(r) \frac{\partial g(r)}{\partial \rho} r^{2} d r
$$

And also to test if the criteria given by Eq. (10) can also be applied for computations of the excess entropy derivative computed up to the second shell we also calculate

$$
\sum_{2}^{(2)}=\left(\frac{\partial s_{2}}{\partial \ln \rho}\right)_{T}=s_{2}^{(2)}-2 \pi \rho^{2} \int_{0}^{r_{2}} \ln g(r) \frac{\partial g(r)}{\partial \rho} r^{2} d r .
$$

Figures 10 and 11 show the behavior of $\sum_{2}$ and of $\sum_{2}^{(2)}$ with the density, respectively, for a fixed temperature for the potentials $A_{3}, B_{3}$, and $C_{3}$. The horizontal lines at $\sum_{2}=0,0.42$, and 1.00 indicate the threshold beyond which there are structural, diffusion, and density anomalies, respectively. The graphs confirm that the density, the diffusion, and the structural anomalous behavior is observed for the potentials $A_{3}$ and $B_{3}$ but not for the potential $C_{3}$, confirming Errington's criteria.

The comparison between Figs. 10 and 11 shows that the derivative of the excess entropy computed up to the second shell is a good approximation for $\sum_{2}$ for all the cases.

This result together with the good agreement between $s_{2}$ and $s_{2}^{(2)}$ supports our surmise that focusing in the first and
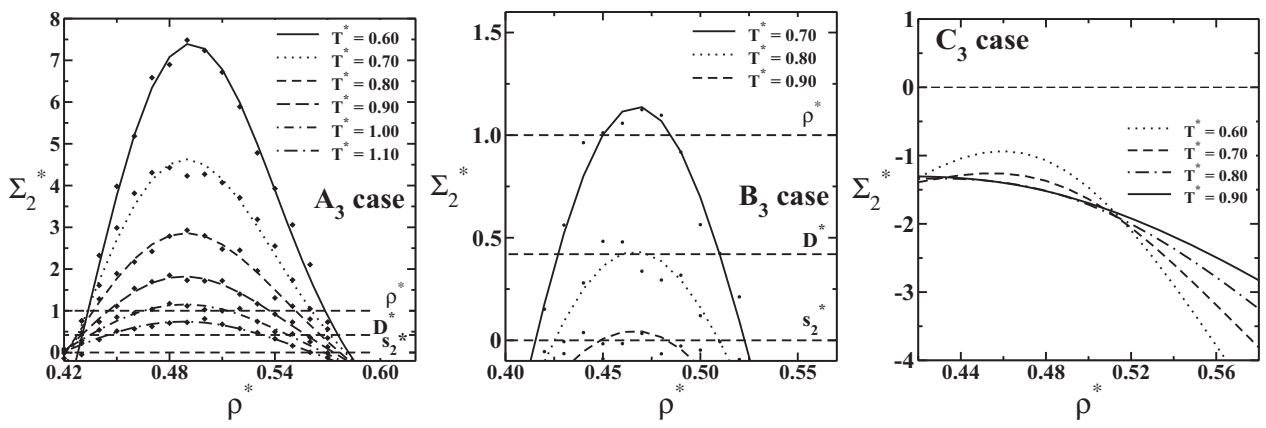

FIG. 10. $\sum_{2}^{(2)}$ versus reduced density for temperatures. 

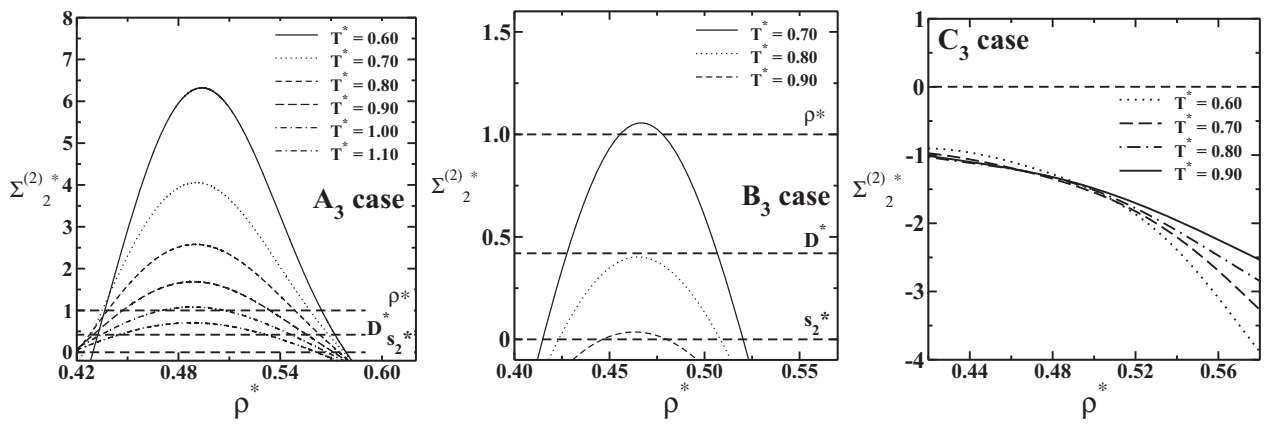

FIG. 11. $\sum_{2}^{(2)}$ versus reduced density computed up to the second coordination shell.

second shell behavior we can understand the mechanism that leads to the anomalous behavior.

\section{CONCLUSIONS}

In this paper, we analyzed three families of potentials characterized by two length scales: a shoulder scale and an attractive scale. We found that when approaching the two scales and keeping the slope between them fixed, the liquid-liquid critical point goes to lower pressures while keeping the critical temperature fixed. This result seems to indicate that the slope of the curve might be related to the critical temperature while the distance between the scales control the critical pressure. This assumption is also supported by another continuous spherical symmetric potential in which the slope was varied. ${ }^{61}$

We also found anomalous behavior in the density, in the diffusion coefficient, in the structural order parameter, and in the excess entropy for all the cases in which the distance between the scales were not too short.

From the behavior of the radial distribution function we did infer that the anomalies are related to particles moving from one scale to the other.

In order to check our assumption the excess entropy and its derivative were computed in two ways: the total value and the value computed by integrating up to the second coordination shell. We found that the behavior obtained by computing these quantities up to the second coordination shell is accurate both for system with and without anomalies. Since the Rosenfeld's parametrization ${ }^{75}$ is just a lower bound, we can say that it is enough to compute $s_{2}$ and $\sum_{2}$ up to the second shell to know if the anomalies are present or not.

\section{ACKNOWLEDGMENTS}

We thank for financial support the Brazilian science agencies $\mathrm{CNPq}$ and Capes. This work is partially supported by CNPq, INCT-FCx.

${ }^{1}$ P. C. Hemmer and G. Stell, Phys. Rev. Lett. 24, 1284 (1970).

${ }^{2}$ G. Stell and P. C. Hemmer, J. Chem. Phys. 56, 4274 (1972).

${ }^{3}$ M. Silbert and W. H. Young, Phys. Lett. A 58, 469 (1976).

${ }^{4}$ E. A. Jagla, J. Chem. Phys. 110, 451 (1999).

${ }^{5}$ E. A. Jagla, J. Chem. Phys. 111, 8980 (1999).

${ }^{6}$ N. B. Wilding and J. E. Magee, Phys. Rev. E 66, 031509 (2002).

${ }^{7}$ P. Camp, Phys. Rev. E 68, 061506 (2003).

${ }^{8}$ P. Vilaseca and G. Franzese, J. Non-Cryst. Solids 357, 419 (2011).

${ }^{9}$ G. S. Kell, J. Chem. Eng. Data 20, 97 (1975).
${ }^{10}$ C. A. Angell, E. D. Finch, and P. Bach, J. Chem. Phys. 65, 3063 (1976).

${ }^{11}$ T. Tsuchiya, J. Phys. Soc. Jpn. 60, 227 (1991).

${ }^{12}$ C. A. Angell, R. D. Bressel, M. Hemmatti, E. J. Sare, and J. C. Tucker, Phys. Chem. Chem. Phys. 2, 1559 (2000).

${ }^{13}$ Z. Y. Yan, S. V. Buldyrev, P. Kumar, N. Giovambattista, and H. E. Stanley, Phys. Rev. E 77, 042201 (2008).

${ }^{14}$ P. Kumar, G. Franzese, and H. E. Stanley, J. Phys.: Condens. Matter 20, 244114 (2008)

${ }^{15}$ A. B. de Oliveira, G. Franzese, P. A. Netz, and M. C. Barbosa, J. Chem. Phys. 128, 064901 (2008)

${ }^{16}$ A. B. de Oliveira, P. A. Netz, and M. C. Barbosa, Europhys. Lett. 85, 36001 (2009).

${ }^{17}$ S. A. Egorov, J. Chem. Phys. 128, 174503 (2008).

${ }^{18}$ N. M. Barraz, Jr., E. Salcedo, and M. C. Barbosa, J. Chem. Phys. 131, 094504 (2009).

${ }^{19}$ R. Waler, Essays of Natural Experiments (Johnson Reprint, New York, 1964).

${ }^{20}$ R. J. Speedy and C. A. Angell, J. Chem. Phys. 65, 851 (1976).

${ }^{21}$ H. Kanno and C. A. Angell, J. Chem. Phys. 70(9), 4008 (1979).

${ }^{22}$ C. A. Angell, M. Oguni, and W. J. Sichina, J. Phys. Chem. 86, 998 (1982).

${ }^{23}$ E. Tombari, C. Ferrari, and G. Salvetti, Chem. Phys. Lett. 300(5-6), 749 (1999).

${ }^{24}$ H. Thurn and J. Ruska, J. Non-Cryst. Solids 22, 331 (1976).

${ }^{25}$ Handbook of Chemistry and Physics, edited by R. C. Weast (CRC Press, Boca Raton, FL, 1984-1985), 65th ed.

${ }^{26}$ G. E. Sauer and L. B. Borst, Science 158, 1567 (1967).

${ }^{27}$ S. J. Kennedy and J. C. Wheeler, J. Chem. Phys. 78, 1523 (1983).

${ }^{28}$ R. Sharma, S. N. Chakraborty, and C. Chakravarty, J. Chem. Phys. 125, 204501 (2006).

${ }^{29}$ M. S. Shell, P. G. Debenedetti, and A. Z. Panagiotopoulos, Phys. Rev. E 66, 011202 (2002).

${ }^{30}$ P. H. Poole, M. Hemmati, and C. A. Angell, Phys. Rev. Lett. 79, 2281 (1997).

${ }^{31}$ S. Sastry and C. A. Angell, Nature Mater. 2, 739 (2003).

${ }^{32}$ F. X. Prielmeier, E. W. Lang, R. J. Speedy, and H.-D. Lüdemann, Phys. Rev. Lett. 59, 1128 (1987)

${ }^{33}$ H. J. C. Berendsen, J. R. Grigera, and T. P. Straatsma, J. Phys. Chem. 91, 6269 (1987)

${ }^{34}$ P. A. Netz, F. W. Starr, H. E. Stanley, and M. C. Barbosa, J. Chem. Phys. 115, 344 (2001).

${ }^{35}$ J. R. Errington and P. G. Debenedetti, Nature (London) 409, 318 (2001).

${ }^{36}$ A. Mudi, C. Chakravarty, and R. Ramaswamy, J. Chem. Phys. 122, 104507 (2005).

${ }^{37}$ J. Mittal, J. R. Errington, and T. M. Truskett, J. Phys. Chem. B 110, 18147 (2006).

${ }^{38}$ P. A. Netz, F. W. Starr, M. C. Barbosa, and H. E. Stanley, Physica A 314, 470 (2002).

${ }^{39}$ P. A. Netz, F. W. Starr, M. C. Barbosa, and H. E. Stanley, J. Mol. Liq. 101, 159 (2002).

${ }^{40}$ T. Morishita, Phys. Rev. E 72, 021201 (2005).

${ }^{41}$ A. Scala, M. R. Sadr-Lahijany, N. Giovambattista, S. V. Buldyrev, and H. E. Stanley, J. Stat. Phys. 100, 97 (2000).

${ }^{42}$ S. V. Buldyrev, G. Franzese, N. Giovambattista, G. Malescio, M. R. SadrLahijany, A. Scala, A. Skibinsky, and H. E. Stanley, Physica A 304, 23 (2002). 
${ }^{43}$ S. V. Buldyrev and H. E. Stanley, Physica A 330, 124 (2003).

${ }^{44}$ A. Skibinsky, S. V. Buldyrev, G. Franzese, G. Malescio, and H. E. Stanley, Phys. Rev. E 69, 061206 (2005).

${ }^{45}$ G. Franzese, G. Malescio, A. Skibinsky, S. V. Buldyrev, and H. E. Stanley, Phys. Rev. E 66, 051206 (2002).

${ }^{46}$ A. Balladares and M. C. Barbosa, J. Phys.: Condens. Matter 16, 8811 (2004).

${ }^{47}$ A. B. de Oliveira and M. C. Barbosa, J. Phys.: Condens. Matter 17, 399 (2005).

${ }^{48}$ V. B. Henriques and M. C. Barbosa, Phys. Rev. E 71, 031504 (2005).

${ }^{49}$ V. B. Henriques, N. Guissoni, M. A. Barbosa, M. Thielo, and M. C. Barbosa, Mol. Phys. 103, 3001 (2005).

${ }^{50}$ E. A. Jagla, Phys. Rev. E 58, 1478 (1998).

${ }^{51}$ S. Maruyama, K. Wakabayashi, and M. A. Oguni, Aip Conf. Proc. 708, 675 (2004).

${ }^{52}$ R. Kurita and H. Tanaka, Science 206, 845 (2004).

${ }^{53}$ L. Xu, P. Kumar, S. V. Buldyrev, S.-H. Chen, P. Poole, F. Sciortino, and H. E. Stanley, Proc. Natl. Acad. Sci. U.S.A. 102, 16558 (2005).

${ }^{54}$ A. B. de Oliveira, P. A. Netz, T. Colla, and M. C. Barbosa, J. Chem. Phys. 124, 084505 (2006).

${ }^{55}$ A. B. de Oliveira, P. A. Netz, T. Colla, and M. C. Barbosa, J. Chem. Phys. 125, 124503 (2006).

${ }^{56}$ A. B. de Oliveira, M. C. Barbosa, and P. A. Netz, Physica A 386, 744 (2007).

${ }^{57}$ A. B. de Oliveira, P. A. Netz, and M. C. Barbosa, Euro. Phys. J. B 64, 481 (2008).

${ }^{58}$ N. V. Gribova, Y. D. Fomin, D. Frenkel, and V. N. Ryzhov, Phys. Rev. E 79, 051202 (2009).
${ }^{59}$ E. Lomba, N. G. Almarza, C. Martin, and C. McBride, J. Chem. Phys. 126, 244510 (2007).

${ }^{60}$ G. Franzese, G. Malescio, A. Skibinsky, S. V. Buldyrev, and H. E. Stanley, Nature (London) 409, 692 (2001).

${ }^{61}$ J. da Silva, E. Salcedo, A. B. Oliveira, and M. C. Barbosa, J. Phys. Chem. 133, 244506 (2010).

${ }^{62}$ Z. Yan, S. V. Buldyrev, N. Giovambattista, P. G. Debenedetti, and H. E. Stanley, Phys. Rev. E 73, 051204 (2006).

${ }^{63}$ P. Vilaseca and G. Franzese, J. Chem. Phys. 133, 084507 (2010).

${ }^{64}$ A. Baranyai and D. J. Evans, Phys. Rev. A 40, 3817 (1989).

${ }^{65}$ J. R. Errington, T. M. Truskett, and J. Mittal, J. Chem. Phys. 125, 244502 (2006).

${ }^{66}$ S. N. Chakraborty and C. Chakravarty, J. Chem. Phys. 124, 014507 (2006).

${ }^{67}$ T. Head-Gordon and F. H. Stillinger, J. Chem. Phys. 98, 3313 (1993).

${ }^{68}$ F. H. Stillinger and T. Head-Gordon, Phys. Rev. E 47, 2484 (1993).

${ }^{69}$ P. G. Debenedetti V. S. Raghavan, and Steven S. Borick, J. Phys. Chem. 95, 4540 (1991).

${ }^{70}$ Z. Yan, S. V. Buldyrev, P. Kumar, N. Giovambattista, P. G. Debenedetti, and H. E. Stanley, Phys. Rev. E 76, 051201 (2007).

${ }^{71}$ M. P. Allen and D. J. Tildesley, Computer Simulations of Liquids, 1st ed. (Claredon, Oxford, 1987).

${ }^{72}$ J. E. Errington, P. G. Debenedetti, and S. Torquato, J. Chem. Phys. 118, 2256 (2003).

${ }^{73}$ P. J. Steinhardt, D. R. Nelson, and M. Ronchetti, Phys. Rev. B 28, 784 (1983).

${ }^{74}$ Z. Yan, S. V. Buldyrev, N. Giovambattista, and H. E. Stanley, Phys. Rev. Lett. 95, 130604 (2005).

${ }^{75}$ Y. Rosenfeld, J. Phys.: Condens. Matter 11, 5415 (1999).

${ }^{76}$ H. E. Stanley, Pramana 53, 53 (1999). 